\title{
A preliminary study on the reliability of physical performance measures in older day-care center clients with dementia.
}

Vince Salazar Thomas

Dartmouth College

Patricia A. Hageman

University of Nebraska Medical Center, phageman@unmc.edu

Tell us how you used this information in this short survey.

Follow this and additional works at: https://digitalcommons.unmc.edu/sahp_pt_articles

Part of the Physical Therapy Commons

\section{Recommended Citation}

Salazar Thomas, Vince and Hageman, Patricia A., "A preliminary study on the reliability of physical performance measures in older day-care center clients with dementia." (2002). Journal Articles: Physical Therapy. 2.

https://digitalcommons.unmc.edu/sahp_pt_articles/2

This Article is brought to you for free and open access by the Physical Therapy at DigitalCommons@UNMC. It has been accepted for inclusion in Journal Articles: Physical Therapy by an authorized administrator of DigitalCommons@UNMC.For more information, please contact digitalcommons@unmc.edu. 


\title{
A Preliminary Study on the Reliability of Physical Performance Measures in Older Day-Care Center Clients With Dementia
}

\author{
Vince Salazar Thomas and Patricia A. Hageman
}

ABSTRACT. Background: Decline in physical functional ability is an intrinsic component of the dementia syndrome. Reductions in muscle mass and strength represent a major factor in the loss of functional ability. Although resistance exercise has been studied as a method for maintaining/recovering function in populations of frail older adults, people with dementia have been systematically excluded because of uncertainty about the reliability of outcome measurements. Objective: The purpose of this study was to determine the test-retest reliability of a battery of established performance-based measures of strength and function among subjects with dementia. Setting: A hospital-affiliated adult day-care facility. Subjects: Twelve of 28 older subjects with dementia of various etiologies were available for two assessments prior to implementation of a resistance-exercise intervention. Methods: Subjects underwent an assessment of lower extremity strength and physical function consisting of two recorded trials of bilateral isometric strength of the knee extensor, hip flexor, and dorsiflexor muscles, as well as hand grip strength; repeated chair stands, evaluation of usual- and maximal safe-gait speed over a 6-m course, and the Timed-Up-and-Go Test. The entire assessment was repeated approximately 1 week later. An average of the trials for each measurement was computed for each of the two assessment periods, and intraclass correlation coefficients (ICCs) for these paired measurements were estimated using STATA. Results: ICCs ranged from .56 for left iliopsoas to .77 for left dorsiflexors among the strength measures whereas measures of function ranged from .80 for number of steps in usual gait to .95 for time of fast gait. Conclusions: Performancebased measures of strength and function can be reliably assessed in older people with dementia, although measures of function appear to be more reliable than measures of strength.

KEYWORDS: Dementia; functional disability; performance-based measures; reliability; resistance exercise

Decline in physical functional abilities is an intrinsic component of the dementia syndrome and the diagnostic determination of dementing illness (American

From the Center for the Aging, Dartmouth Medical School, Hanover, New Hampshire, US (V. S. Thomas, PhD), and Division of Physical Therapy Education, School of Allied Health, University of Nebraska Medical Center, Omaha, Nebraska, US (P. A. Hageman, PhD, PT).
Psychiatric Association, 1994; World Health Organization, 1992). For patients as well as their caregivers, functional decline may be the most problematic

Offprints. Requests for offprints should be directed to Dr. Vince S. Thomas, 102A Butler, Center for the Aging, Dartmouth Medical School, Hanover, NH 03755, US. email: vince.s.thomas@dartmouth.edu 
aspect of dementia because loss of the ability to function independently leads to an increase in the need for care and increases the risk of institutionalization, and such formal and informal care accounts for the majority of diseaserelated costs (Kinosian et al., 2000; Taylor \& Sloan, 2000). Deconditioning and mobility limitations appear to frequently be premature and unnecessary initial sequelae of a dementia diagnosis due, at least in part, to an underestimation of residual abilities and decreased expectations of the patient by carers ("Alzheimer's Treatments That Work Now," 1998). Because treatment options to abate the cognitive decline related to dementia are currently limited, attention to concurrent physical impairments is appropriate. Although resistance exercise has been increasingly studied as a method for reducing impairments and recovering function in frail elderly populations (Singh, 1998; Singh et al., 1999; Yarasheski et al., 1999), people with dementia have been systematically excluded from such studies because of uncertainty about the reliability of outcome measurements (Tappen et al., 1997). The concern is not unfounded, because dementia produces a progressive deterioration in memory, judgment, and attention, and the concomitant manifestation of aphasia and apraxia (American Psychiatric Association, 1994). Additionally, impaired balance, diminished concentration ability, and apprehension to unfamiliar devices may impede performance-based measurements (Brill et al., 1995). The purpose of this study was to examine the test-retest reliability of a battery of established performance-based measures of strength and function among subjects with dementia.

\section{METHODS}

\section{Subjects}

As part of a pilot trial of resistance exercise, 28 subjects with dementia were recruited from a defined population of elderly day-care users with an established diagnosis of dementia (of various etiologies), in attendance at one of two adult day-care facilities operated by a local hospital. Subjects were excluded from the study if they had experienced a heart attack or stroke within the last 6 months or if they had a condition that might be worsened by the exercise. Subjects were also excluded if they were unable to independently ambulate with or without an assistive device for a distance of 10 meters. Written informed consent was obtained from all subjects or, as necessary, from their legal guardians, in accordance with Institutional Review Board guidelines of Alegent Health Center and the University of Nebraska Medical Center.

\section{Procedure}

Measurement procedures adapted from the protocols used in various studies of both institutional and communitydwelling populations (e.g., Gerety et al., 1993; Guralnik et al., 1995), and modified to fit time and population constraints, were used in conducting the assessment of lower extremity strength and function. The assessment was repeated for 12 subjects, on average, 7 days after the initial assessment. Reassessment for the remaining 16 subjects was not performed due to the inability to schedule the retesting within a 6-to 9-day window after the initial assessment.

\section{Performance-Based Measures of Muscle Strength}

Maximum strength of the knee extensor, hip flexor, and dorsiflexor muscles was 
assessed bilaterally using a Microfet2 manual muscle tester (Hogan Health Industries, Inc., Draper, UT). The Microfet2 is a force evaluation and testing system used in hand-held dynamometry. For knee and hip flexor assessment, the subject was seated in a hard chair (on a platform) with knees and hips flexed to $90^{\circ}$ and arms crossed. For knee extensor testing, the subject was asked to hold the knee in a position at approximately $80^{\circ}$ of knee flexion with the placement of the dynamometer two fingers above the medial malleoli (knee extension). For hip flexor testing, subjects were asked to lift the thigh above the chair while the examiner placed the dynamometer 2 inches proximal to the femoral condyles at the distal thigh. Ankle dorsiflexion strength was measured with the subject seated with the leg fully extended on an 8-inch footrest and the dynamometer placed proximal to the first metatarsal head of the foot. Subjects were asked to push as hard as they could against the dynamometer. Strength was recorded as the peak force, expressed in kilograms force, that the examiner had to apply to break the isometric contraction, moving the subject's leg in the direction opposite to the voluntary movement. During the test, the subject was allowed to place his/her hands on the front edge of the chair seat but not to lean backward. Subjects completed up to five trials on each side for each muscle group-fewer if two measurements within $10 \%$ of each other were recorded. The assessments were attempted only in subjects who could first perform the movements against gravity alone.

Isometric hand-grip strength was also measured bilaterally using a Jamar hand-held dynamometer (Sammons
Preston, Inc., Bolingbrook, IL). Grip strength, which is a general measure of upper extremity strength and is important for all functional activities involving the lifting of objects or manipulation of tools, represents, together with knee extensor, hip flexor, and dorsiflexor muscles, the core of muscle groups that have been shown to be important for performing daily activities (Hunter et al., 1995), such as walking and transferring (lower extremity muscles) and dressing, picking up objects, and even eating (grip strength).

\section{Performance-Based Measures of Function}

Lower extremity function was also evaluated using a modification of the testing batteries from previous studies (e.g., Gerety et al., 1993; Guralnik et al., 1995). This consisted of three performancebased tests that assess (a) walking speed, (b) time to rise from a chair and sit down five times (chair stands), and (c) standing balance. Walking speed was assessed by having the participant walk at his/her usual pace over a $6-\mathrm{m}$ course, and then reassessed at a maximal safe pace. Participants were instructed to stand with both feet touching the starting line and to start walking after a specific verbal command. Timing began when the command was given, and the time needed to complete the entire distance was recorded. The average of two walks was used to compute a measure of walking speed. The number of steps was also recorded and was used to determine stride length. Aids were discouraged for this test, but permitted if necessary. The test of repeated chair stands was performed using an armed, straight-back chair with a seat approximately $45 \mathrm{~cm}$ 
high at the front edge. Participants were first asked to stand from a sitting position without using their arms. If they were able to perform this activity, they were then asked to stand up and sit down five times as quickly as possible. The time to complete the entire task was recorded. Standing balance was assessed by asking subjects to maintain balance in three positions characterized by a progressive narrowing of the base of support.

The Timed-Up-And-Go (TUG; Posiadlo \& Richardson, 1991) test was also used, in order to measure the time in seconds that an individual required to stand up from the chair $45 \mathrm{~cm}$ high, walk 3 meters, return, and sit down (Figure 1). Subjects were permitted to use assistive devices if needed. Each subject completed two trials of this test. For all sub-

\section{- Muscle Strength}

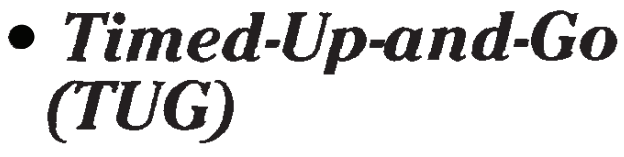

- Sit-to-stand

- Usual \& Fast Gait

- Other measures: standing balance, somatosensory discrimination jects, there was nearly total consistency in which examiners administered each test and recorded measurements.

\section{Other Study Measurements}

Cognitive function was determined by application of the Folstein Mini-Mental State Examination (MMSE; Folstein et al., 1975). Functional ability was assessed using a modified version of the Health Interview Survey Supplement on Aging (Fried et al., 1994) questionnaire administered to primary caregivers and to capable subjects. Somatosensory discrimination was evaluated by light touch discrimination using the SemmesWeinstein monofilaments and performed on the bare foot of the dominant leg with the subject sitting with the foot propped

- Maximum isometric strength of the knee extensor, hip flexor and dorsiflexor muscles

- Isometric hand grip strength

- Time to stand from an armed, straight-back chair $45 \mathrm{~cm}$ high, walk $3 \mathrm{~m}$ at comfortable pace (using typical assistive device), turn, return to chair and sit down

- Five repeated chair stands from a sitting position with arms folded

-Walking speed assessed at (1) usual and (2) fast pace over a $6 \mathrm{~m}$ course

Figure 1. Summary of performance-based measures. 
up on a chair. Analyses of performance on these measurements as well as that of standing balance will be reported on elsewhere.

\section{Statistical Analysis}

An average of the trials for each measurement was computed for each of the two assessments, and intraclass correlations (ICCs) for these paired measurements were estimated based on the variance component using STATA (StataCorp., 1997). According to guidelines proposed by Fleiss (1986), ICCs ranging from 0.0 to 0.40 can be considered to be poor, whereas values ranging from 0.41 to 0.75 can be considered as fair to good, and values from 0.75 to 1.00 should be considered to be excellent.

\section{RESULTS}

Among the subjects with test-retest data, the average MMSE score was 16.9 \pm 7.3 . The average age was $80.5 \pm 6.2$, while the average height, weight, and body mass index were, respectively, $159.4 \pm 9.0 \mathrm{~cm}, 69.4 \pm 17.8 \mathrm{~kg}$, and $27.0 \pm$ $5.3 \mathrm{~kg} / \mathrm{m}^{2}$. All 12 subjects were female and 7 of the subjects were non-White (either African American or from the Caribbean Basin).

As displayed in Table 1, the ICCs for bilateral lower extremity strength were fair to good, ranging from .56 for left iliopsoas to .77 for left dorsiflexor. The ICC for left grip strength was .70 whereas that for the right was slightly lower, at .68. Meanwhile, the ICCs for lower extremity dynamic function were excellent, ranging from .80 for steps taken in 6-m gait at usual speed to .94 for the repeated sit-to-stand measure.

\section{DISCUSSION}

We found that the test-retest reliability of the strength measures in this population were good for the dorsiflexors and knee extensors, and fair for the hip flexors. The relatively low coefficient estimates for testing of the hip flexors suggest, in turn, a lack in the validity of this measure in this sample. This finding is not surprising given that the examiners found the subjects to have difficulty understanding this test, despite repeated instructions and demonstrations by the examiners. Meanwhile, the better estimates of reliability for assessment of strength of the dorsiflexors and knee extensors reflect strength estimates within the normative range for asymptomatic 70-79-year-olds based on testing with a similar protocol (Andrews et al., 1996). These results can be contrasted to estimates derived from cognitively intact populations, where the test-retest reliability of hand-held dynamometry has been reported to be high, with ICCs ranging from .91 to .98 depending on the muscle region (Andrews et al., 1996; Ferruci et al., 1997).

The reliability estimates of lower extremity function were generally excellent. The subjects in our study were able to follow the standard TUG test instructions; whereas in Tappen and colleagues' (1997) study of 33 subjects with moderate to severe Alzheimer's disease institutionalized in long-term facilities, the researchers were forced to modify the TUG because many individuals in their study had difficulty understanding and following verbal commands. Minor modifications to the assessment protocol were also necessary in our study: e.g., it was 
TABLE 1. Test-Retest Reliabilities

\begin{tabular}{|c|c|c|c|c|c|c|}
\hline \multirow{2}{*}{$\begin{array}{l}\text { Performance-Based } \\
\text { Measures }\end{array}$} & \multicolumn{2}{|l|}{ Test 1} & \multicolumn{2}{|l|}{ Test 2} & \multirow{2}{*}{$\begin{array}{c}\text { Intraclass Correlation } \\
\text { Coefficient }\end{array}$} & \multirow[b]{2}{*}{$n$} \\
\hline & $M(S D)$ & $n$ & $M(S D)$ & $n$ & & \\
\hline Left iliopsoas & $35.09(14.59)$ & 11 & $34.82(8.18)$ & 11 & .56 & 11 \\
\hline Right iliopsoas & $37.63(16.17)$ & 12 & $38.04(10.24)$ & 12 & .63 & 12 \\
\hline Left quadriceps & $24.79(9.85)$ & 12 & $29.96(8.29)$ & 12 & .62 & 12 \\
\hline Right quadriceps & $27.75(10.17)$ & 12 & $33.21(9.79)$ & 12 & .71 & 12 \\
\hline Left dorsiflexor & $42.5 \quad(21.12)$ & 11 & $43.45(15.22)$ & 10 & .77 & 10 \\
\hline Right dorsiflexor & $40.79(20.36)$ & 12 & $47.41(11.91)$ & 11 & .63 & 11 \\
\hline Left grip & $14.24(2.61)$ & 11 & $15.63(5.20)$ & 9 & .70 & 9 \\
\hline Right grip & $14.92(6.29)$ & 12 & $18.3(6.17)$ & 10 & .68 & 10 \\
\hline Sit-to-stand & $23.65(9.49)$ & 12 & $21.68(9.07)$ & 10 & .94 & 10 \\
\hline Time, usual gait & $12.07(5.87)$ & 12 & $11.88(6.48)$ & 10 & .92 & 10 \\
\hline Steps, usual gait & $16.00(3.48)$ & 12 & $14.90(5.60)$ & 10 & .80 & 10 \\
\hline Time, fast gait & $8.42(4.48)$ & 10 & $7.21(4.15)$ & 9 & .95 & 9 \\
\hline Steps, fast gait & $13.9(3.71)$ & 10 & $12.83(3.17)$ & 9 & .90 & 9 \\
\hline Timed-Up-and-Go & $29.56(20.55)$ & 12 & $21.35(11.41)$ & 9 & .87 & 9 \\
\hline
\end{tabular}

often necessary to repeatedly demonstrate a procedure; timing/recording often began when the subject initiated the trial; subtle guidance was often used, either verbally or in the form of pointing or directing. "Turning" presented the only systematic problem for subjects, whereas sustained contraction/extension was problematic for a few subjects.

Our results affirm the contention that many physical performance measures can be reliably employed in testing individuals with progressed dementia (Brill et al., 1995; Tappen et al., 1997). In contrast to past reports, however, we find that significant modification to test protocols may be unnecessary, rendering the test results more comparable to those derived from nondemented older populations. The accumulation of evidence demonstrating the reliability of outcome measurement among demented subjects should lead to the cessation of systematic exclusion of demented people from trials of exercise rehabilita- tion. Such studies, which have been found to be highly effective among cognitively intact frail elderly persons, may represent important mechanisms for the treatment of concomitant physical impairments resultant from the dementing illness process or from nondementia illnesses.

\section{REFERENCES}

Alzheimer's treatments that work now. (1998). Science, 282, 1030-1032.

American Psychiatric Association. (1994). Diagnostic and statistical manual of mental disorders (4th ed.). Washington, DC: Author.

Andrews, A. W., Thomas, M. W., \& Bohannon, R. W. (1996). Normative values for isometric muscle force measurements obtained with hand-held dynamometers. Physical Therapy, 76, 248-259.

Brill, P. A., Drimmer, A. M., Morgan, L. A., \& Gordon, N. F. (1995). The feasibility of conducting strength and flexibility programs 
for elderly nursing home residents with dementia. The Gerontologist, 35, 263-266.

Ferruci, L., Guralnick, J. M., Buchner, D., Kasper, J., Lamb, S. E., et al. (1997). Departures from linearity in the relationship between measures of muscular strength and physical performance of the lower extremities: The Women's Health and Aging Study. Journal of Gerontology, 52A, M275-M285.

Fleiss, J. L. (1986). The design and analysis of clinical experiments. New York: Wiley.

Folstein, M. F., Folstein, S. E., \& McHugh, P. R. (1975). Mini-Mental State: A practical method for grading the cognitive state of patients for the clinician. Journal of Psychiatric Research, 12, 189-198.

Fried, L. P., Ettinger, W. H., Lind, B., et al. (1994). Physical disability in older adults: A physiological approach. Journal of Clinical Epidemiology, 47, 747-760.

Gerety, M. B., Mulrow, C. D., Tuley, M. R., et al. (1993). Development and validation of a physical performance instrument for the functionally impaired elderly: The Physical Disability Index (PDI). Journal of Gerontology Medical Science, 48, M33-M38.

Guralnik, J. M., Fried, L. P., Simonsick, E. M., Kasper, J. D., \& Lafferty, M. E. (1995). The Women's Health and Aging Study: Health and social characteristics of older women with disability (NIH Publication No. 95-4009). Bethesda, MD: National Institute on Aging.

Hunter, G. R., Treuth, M. S., Weinster, R. L., et al. (1995). The effects of strength conditioning on older women's ability to perform daily tasks. Journal of the American Geriatrics Society, 43, 756-760.

Kinosian, B. P., Stallard, E., Lee, J. H., et al. (2000). Predicting 10-year care requirements for older people with suspected Alzheimer's disease. Journal of the American Geriatrics Society, 48, 631-638.

Posiadlo, D., \& Richardson, S. (1991). The timed "Up \& Go": A test of basic functional mobility for frail elderly persons. Journal of the American Geriatrics Society, 39, 142-148.
Singh, M. A. (1998). Combined exercise and dietary intervention to optimize body composition in aging. Annals of the New York Academy of Sciences, 854, 378-393.

Singh, M. A., Ding, W., Manfredi, T. J., et al. (1999). Insulin-like growth factor I in skeletal muscle after weight-lifting exercise in frail elders. American Journal of Physiology, 277, E135-E143.

StataCorp. (1997). Stata statistical software: Release 5.0. College Station,TX: Stata Corporation.

Tappen, R. M., Roach, K. E., Buchner, D., Barry, C., \& Edelstein, J. (1997). Reliability of physical performance measures in nursing home residents with Alzheimer's disease. Journal of Gerontology Medical Science, 52A, M52-M55.

Taylor, D. H., Jr., \& Sloan, F. A. (2000). How much do persons with Alzheimer's disease cost Medicare? Journal of the American Geriatrics Society, 48, 639-646.

World Health Organization. (1992). International statistical classification of diseases and related health problems, 10th revision (ICD-10). Chapter V, categories F00-F99. Mental, behavioural and developmental disorders: Clinical description and diagnostic guidelines. Geneva, Switzerland: Author.

Yarasheski, K. E., Pak-Loduca, J., Hasten, D. L., et al. (1999). Resistance exercise training increases mixed muscle protein synthesis rate in frail women and men $>/=76 \mathrm{yr}$ old. American Journal of Physiology, 277, E118-E125.

Acknowledgment. This work was supported by a Vada Kinman Oldfield Scholar in Alzheimer's Disease Research Award and a Claude D. Pepper Older Americans Independence Center Grant to V. S. Thomas. We are grateful to Jill Brown, Ryan McCabe, Jeff Arnold, and Amberlyn Divis for their efforts in helping to conduct the study and to the staff and clients of the McAuley Bergen Adult Daycare Centers for their gracious help and willing participation. 